QCD Evolution Workshop 2014

International Journal of Modern Physics: Conference Series

Vol. 37 (2015) 1560066 (8 pages)

(C) The Author

DOI: $10.1142 /$ S2010194515600666

\title{
Recent Results on the Measurement of Fragmentation Functions in $e^{+} e^{-}$Annihilation
}

\author{
Anselm Vossen \\ Center for Exploration of Energy and Matter, Indiana University, \\ 2401 Milo B Sampson Lane Bloomington, Indiana 47408, USA \\ Published 25 February 2015
}

\begin{abstract}
The precise knowledge of fragmentation functions is needed to extract information about nucleon structure from semi-inclusive measurements. They themselves are also important objects to study the properties of nonperturbative QCD. This contribution will summarize recent results from measurements at the B-factories Belle and BaBar.
\end{abstract}

\section{Introduction}

Fragmentation functions (FFs) describe the probability that a quark $q$ fragments into a hadron $h$. Similarly to parton distribution functions, it is customary to name them $D, G, H$, depending on the initial quark being unpolarized, longitudinally polarized and transversely polarized, respectively. We also add a subscript to indicate the twist of the function, but this contribution only discusses leading twist FFs, so that the unpolarized fragmentation function will be denoted $D_{1}^{q}(z)$. Here $z$ denotes the fraction of the quark momentum the hadron is carrying. If the fragmentation function vanishes when integrated over the intrinsic transverse momentum $k_{T}$, we add $\perp$ to the fragmentation function, so that e.g. the Collins fragmentation functions, which describes the transverse polarization dependent fragmentation is denoted $H_{1}^{q, \perp}\left(z, k_{T}\right)$, where $k_{T}$ is a two dimensional vector. Obviously transverse momentum dependence does not have to be symmetric, in the example of the Collins function, the transverse momentum generated in the fragmentation process is dependent on the parent quark polarization, which makes this function so important as a quark polarimeter in Semi-Inclusive Deep-Inelastic-Scattering (SIDIS). This gives already one important motivation for the precision measurement of fragmentation functions: Given factorization and universality, SIDIS cross-sections can be decomposed into distribution functions, describing quark and gluon dynamics, a hard scattering cross-section which can be computed in perturbative QCD and the fragmentation functions. So in order to extract the structure of the nucleon from SIDIS,

This is an Open Access article published by World Scientific Publishing Company. It is distributed under the terms of the Creative Commons Attribution 3.0 (CC-BY) License. Further distribution of this work is permitted, provided the original work is properly cited. 
such as flavor dependent distribution functions or intrinsic transverse momentum of quarks inside the nucleon, one has to know the fragmentation functions. Recently the class of chiral-odd fragmentation functions received increased attention because they are needed to access chiral-odd parton distribution functions. But in addition to the utility of fragmentation functions as a necessary ingredient to extract nucleon structure from experimental results, FFs are also fundamental quantities of QCD. Similar to parton distribution functions, fragmentation functions FFs, can be written as correlators which are nonlocal matrix elements of partonic field operators (following [1]):

$$
\Xi\left(\kappa, P_{h}, S_{h}\right)=\sum_{X} \int \frac{d^{3} \vec{P}_{X}}{(2 \pi)^{3} 2 E_{X}} \int d^{4} \xi e^{i \kappa \cdot \xi}\left\langle 0\left|\Psi_{i}(\xi)\right| P_{h} S_{h}, X\right\rangle\left\langle P_{h}, S_{h}, X\left|\bar{\Psi}_{j}(0)\right| 0\right\rangle,
$$

where the quark fields operator on the vacuum instead of the nucleon. Here, $P_{h}, S_{h}$ are the momentum and spin quantum numbers of the final state hadron, $\kappa$ the quark momentum Therefore we can gain an understanding of topics such as QCD evolution from studying FFs that is only valid for our theory of the nuclear structure. One of the main differences between parton distribution functions and fragmentation function is obviously that for FFs the initial and final state differ. This means that fragmentation functions cannot be computed on the lattice and have to be measured. While fragmentation functions can be extracted from semi-inclusive measurements in SIDIS or $p+p$ scattering, the cleanest way to access them is in $e^{+} e^{-}$annihilation where a quark-antiquark pair is created that then fragments into hadrons.

\section{Experiments}

Fragmentation functions have been measured over a wide range of energies.. Data is available from center of mass energies (CMEs) of the $Z$-mass at LEP and SLAC down to about $9 \mathrm{GeV}$ at the Argus experiment. However the advent of the Bfactories was a game changer for the study of FFs in $e^{+}-e^{-}$. With a world record instantaneous luminosity of $2.11 \times 10^{34}$ reached e.g., at Belle, they collected more than three orders of magnitude of the integrated luminosity than the LEP experiments. B-factories measure at or near the $\Upsilon(4 S)$ resonance, therefore near a CME of $10.58 \mathrm{GeV}$. This brings the additional advantage, that the measurement is done at a scale much closer to the SIDIS experiments than at e.g., LEP experiments. On the ohter hand it should be mentioned though that compared to SIDIS or $p+p$, in $e^{+} e^{-}$, all quark flavors with masses much smaller than the center of mass energy, are created with relative fractions proportional to their charges squared. This means in particular, that for measurements at B-factories that are described in these proceedings, up to $40 \%$ of the events originate from charm production depending on the final state.

The first generation of B-factories consisted of the Belle and BaBar experiments which were located at KEK and SLAC, respectively. ${ }^{2}$ Belle collected an overall 
integrated luminosity (on- and off-resonance) of more than $1 \mathrm{ab}^{-1}$, which corresponds to about $2 \cdot 10^{9}$ events, and Babar collected about half that. Both experiments are instrumented similarly. Both are situated at the interaction region of asymmetric $e^{+} e^{-}$beams, with positron beam energies of 3.5 and $3.1 \mathrm{GeV}$, respectively, and electron beam energies of 8 and $9 \mathrm{GeV}$ respectively. A setup that boosts B mesons, that are produced at rest in the CMS and allows to measure their decay lengths. Both detectors are typical collider detectors, with the exception that their detector configuration is aimed at being symmetric in the CMS, not the Lab system. Both have vertex trackers, a central drift chamber for tracking and PID using $\mathrm{dE} / \mathrm{dx}$ and an electromagnetic calorimeter as well as a muon identification system using resistive plate chambers. The main difference is in the charged particle ID. Babar utilizes a Detector for Internally reflected Cherenkov light (DIRC) in the central region, in addition to $\mathrm{dE} / \mathrm{dx}$ information, whereas Belle uses a combination of time-of-flight, aerogel Cherenkov counters and $\mathrm{dE} / \mathrm{dx}$ measurements for PID. PID performance varies over the momentum range of the particles and details can be found in Ref. [2]. In general, kaon purities are about $85 \%$ at both experiments with Babar reaching much higher purities at lower momenta, and pion purities are over $90 \%$.

\section{Unpolarized Cross Section Measurements}

The spin averaged fragmentation functions $D_{1}^{q}$ can be extracted from the crosssection for hadron production. Both, Belle and Babar recently published differential cross-section measurements for identified pion and kaon production. ${ }^{3,4}$ Here, I will describe the Belle measurement as BaBar followed a very similar scheme. Belle used a majority of the full off-resonance dataset $\left(68 \mathrm{fb}^{-1}\right)$ for the analysis, whereas BaBar only used a fraction of their data $\left(0.91 \mathrm{fb}^{-1}\right)$ leading to significantly larger error bars. For both experiments the precision at high $z$ is dominated by the systematic errors. To extract the cross-section the approach outlined in Eq. (2)

$$
\frac{d \sigma_{i}}{d z}=\frac{1}{\mathrm{~L}_{\text {tot }}} \epsilon_{\text {joint }}^{i}(z) e_{\mathrm{ISR} / \mathrm{FSR}}^{i}(z) S_{z z_{m}}^{-1} \epsilon^{i} \operatorname{impu}\left(z_{m}\right) P_{i j}^{-1} N^{j, \mathrm{raw}}\left(z_{m}\right)
$$

was taken. Here $N^{j \text {,raw }}$ are the counts for an identified particle $j$. At Belle we used particles identified as charged electrons, muons, kaons, pions or protons by a maximum likelihood method that takes the PID detector signals as input. Then $P_{i j}^{-1}$ is the inverse of the PID correction matrix, which the probability that a particle $j$ is actually a particle $i$. Since we are only interested in identified pion and kaon crosssections, $i \in\{\pi, K\}$. The PID probability matrix is extracted from data from known decays with small backgrounds. As an example the $D^{*}$ decay into a fast and a slow pion and kaon can be used to determine the misidentification ratios of pions and kaons. Since these probabilities will depend on the polar angle in the lab system and the particle momenta, one matrix is build for each bin in $\left(\theta_{\text {lab }}, p\right)$. Where insufficient statistics are available, extrapolations between data and simulations are used. The factor $\epsilon_{\mathrm{impu}}$ gives the dilution due to other processes than the $e^{+}+e^{-} \rightarrow\{u, d, s, c\}$, 
in particular $e^{+}+e^{-} \rightarrow \gamma \gamma$. The factor $\epsilon_{\text {joint }}$ compiles corrections from decay-inflight, detector interactions (particles lost/produced) as well as detector efficiencies, $\epsilon_{\text {ISR/FSR }}^{i}$ describes the correction due to initial and final state radiation and $S_{z z_{m}}^{-1}$ is the inverse of the smearing matrix in the variable $z$. For final and initial state radiation corrections, events were removed when more than $0.5 \%$ of the initial center of mass energy was lost. This was motivated by the relative precision of next-toleading order QCD DGLAP evolution between different center-of-mass energies. This precision is estimated to be around $5 \%$, therefore the allowed change in CME was chosen a magnitude smaller. All corrections with the exception of the PID matrices where extracted from simulations which match the Belle data very well. ${ }^{2}$

The results obtained on the differential cross-sections by Belle and BaBar agree quite well in general and with global data on fragmentation functions. ${ }^{5}$

\section{Collins Effect for Identified Kaons}

Another recent highlight from Belle has been the release of the results for Collins asymmetries for identified charged hadron pairs including kaons. The Collins fragmentation function ${ }^{6} H_{1}^{q, \perp}$ is a chiral-odd $\mathrm{FF}$ that also depends on the intrinsic transverse momentum in the fragmentation. The knowledge of the Collins FF is important since it can serve as a quark polarization analyzer due to the dependency of the intrinsic transverse momentum in the fragmentation on the initial quark spin. It can therefore be used to extract chiral odd parton distribution functions like transversity and the Boer Mulders functions. ${ }^{1}$ In particular recent results ${ }^{7}$ which report large asymmetries sensitive to the Boer-Mulders function for kaons in SIDIS experiments motivate the measurement of the kaon Collins FF. At Belle, this process is measured in $e^{+}+e^{-} \rightarrow\left(q \rightarrow h_{1} X\right)\left(\bar{q} \rightarrow h_{2} X\right)$. Since at Belle the beams are unpolarized and the Collins FF is chiral odd, this effect cannot be measured in $e^{+}+e^{-} \rightarrow\left(q \rightarrow h_{1} X\right)$. Instead the spin correlation of the $q \bar{q}$ pair leads to a $\sin \left(\Phi_{1}+\Phi_{2}\right)$ correlation of the produced hadron pair $h_{1}, h_{2}$. The $\Phi_{i}$ are the azimuthal angles of $h_{i}$ around the thrust axis in the event and $h_{1}, h_{2}$ are back-to-back. The challenge in this analysis, compared to extracting the Collins effect in SIDIS, is that one cannot use spin asymmetries to cancel detector effects and other systematics. For this reason, the analysis is restricted to the central area of the detector, where the acceptance is smooth enough to not incur significant false asymmetries. The PID study described in sec. 3 was used to unfold pion and kaon PIDs.

Results have been presented already at DIS 2014 [8] and show significant asymmetries for kaon-kaon pairs which are of similar magnitude as the $\pi-\pi$ correlations previously measured by Belle and BaBar. ${ }^{9,10}$ In contrast, asymmetries for $\pi-K$ are significantly smaller and even compatible with zero given current systematic and statistical error bars. However, an extraction of the actual light quark kaon FF from these results needs a more sophisticated global analysis since the asymmetries are linear combinations of all combinations of favored and unfavored fragmentation functions leading to the measured final state. In addition there is a significant 
contribution by charm quarks, for likesign $K K$ and $\pi K$ pairs which amounts on average to about $50 \%$.

\section{Di-hadron Fragmentation}

Di-hadron FFs describe the fragmentation of a parton into a pair of hadrons: $q \rightarrow$ $h_{1} h_{2}+X$. Since the final state has more degrees of freedom than is the case for single hadron fragmentation, one can measure transverse spin dependent asymmetries in di-hadron production without detecting the intrinsic transverse momentum. This allows one to treat these asymmetries in a collinear framework. ${ }^{11}$ The di-hadron fragmentation function into charged pion pairs was already measured by Belle ${ }^{12}$ and lead to the first extraction of transversity in a collinear picture. ${ }^{13}$ However, the full cross-section for di-hadron production in electron-positron annihilation contains additional modulation which might be present. Following [11], the correlator $\Delta$ is proportional to

$$
\begin{aligned}
\left.\int d k^{+} \Delta\left(\kappa ;, P_{h}, R\right)\right|_{\kappa^{-}=P_{h}^{-} / z k_{T} \propto} & \left\{D_{1}^{q}\left(z, \xi, \kappa_{T}^{2}, R_{T}^{2}, \kappa_{T} \cdot R_{t}\right) \hbar_{-}\right. \\
& -G_{1}^{\perp, q}\left(z, \xi, \kappa_{T}^{2}, R_{T}^{2}, \kappa_{T} \cdot R_{t}\right) \frac{\epsilon_{\mu \nu \rho \sigma} \gamma^{\mu} n_{-}^{\nu} \kappa_{T}^{\rho} R_{T}^{\sigma}}{M_{1} M_{2}} \gamma_{5} \\
& +H_{1}^{\varangle, q}\left(z, \xi, \kappa_{T}^{2}, R_{T}^{2}, \kappa_{T} \cdot R_{T}\right) \frac{\sigma_{\mu \nu} R_{T}^{\mu} n_{-}^{\nu}}{M_{1}+M_{2}} \\
& \left.+H_{1}^{\perp q}\left(z, \xi, \kappa_{T}^{2}, R_{T}^{2}, \kappa_{T} \cdot R_{T}\right) \frac{\sigma_{\mu \nu} k_{T}^{\mu} n_{i}^{\nu}}{M_{1}+M_{2}}\right\} .
\end{aligned}
$$

Here $\kappa$ is again the outgoing quark momentum, $P_{h}$ the sum of the two hadron momenta and $R$ the difference vector, $M_{1}, M_{2}$ the masses of the outgoing hadrons, $n_{-}$is a lightlike vector along the minus direction and $\xi$ gives the fraction of the momentum carried by $h_{1}\left(\xi=\frac{P_{1}^{-}}{P_{1}^{-}-P_{2}^{-}}\right)$. The term with the function $H_{1}^{\varangle, q}$ describes the correlation of the transverse polarization with the di-hadron plane. Similar to the measurement of the Collins FF, di-hadron asymmetries that dependent on the initial quark polarization can only be measured in the correlation of di-hadron pairs. Therefore, not only $\Phi_{R}$ is introduced, but also $\Phi_{\bar{R}}$ for the same vector on the antiquark side and the correlations corresponding to the spin dependent terms in eq. 3 in the angles $\Phi_{R, \bar{R}}$ are shown in Eqs. (4) and (5). The first correlation corresponding to the interference fragmentation function (IFF) $H_{1}^{\varangle}$ has already been measured by Belle: ${ }^{12}$

$$
\left\langle\cos \left(\Phi_{R}+\Phi_{\bar{R}}\right)\right\rangle=\sum_{q, \bar{q}} e_{q}^{2} \frac{3 \alpha^{2}}{Q^{2}} \frac{z^{2} \bar{z}^{2} B(y)}{\left(M_{1}+M_{2}\right)\left(\bar{M}_{1}+\bar{M}_{2}\right)} H_{1(R)}^{\varangle q}\left(z, M_{h}^{2}\right) \bar{H}_{r,(R)}^{\varangle q}\left(\bar{z}, \bar{M}_{h}^{2}\right) .
$$

However, the correlator contains more terms. Obviously there is the unpolarized part $D_{1}^{q}$, but there is also the function $G_{1}^{\perp, q}$. From the correlator, one can see 
that it parameterizes the helicity dependent correlation of the intrinsic transverse momentum generated in the fragmentation with the di-hadron plane. Similar to the IFF, it also leads to a correlation in the azimuthal angles of the di-hadron planes as given by:

$$
\left\langle\cos \left(2\left(\Phi_{R}-\Phi_{\bar{R}}\right)\right)\right\rangle=\sum_{q, \bar{q}} e_{a}^{2} \frac{3 \alpha^{2}}{2 Q^{2}} z^{2} \bar{z}^{2} A(y) \frac{1}{M_{1} M_{2} \bar{M}_{1} \bar{M}_{2}} G_{1}^{\perp, q}\left(z, M_{h}^{2}\right) \bar{G}_{1}^{\perp, q}\left(\bar{z}, \bar{M}_{h}^{2}\right) .
$$

One significant difference compared to $H_{1}^{\varangle}$, is that $G_{1}^{\perp}$ parametrizes a correlation that vanishes, if the intrinsic transverse momentum is integrated out. This is evident from the fact that the term in the correlator explicitly depends on $\kappa_{T}$ and is reflected in the $\perp$ superscript of the function. Measuring a significant correlation "would be a crucial test of the dihadron TMD FF formalism" [14] since without the intrinsic $k_{T}$, the asymmetry would not be allowed. ${ }^{15,16}$ Since the intrinsic $k_{T}$ plays a similar role in the Boer-Mulders function which was proposed to explain the violation of the Lam-Tung relation, a confirmation of the existence of $G_{1}^{\perp}$ would support this approach. Additionally to the two asymmetries relating to $G_{1}^{\perp}$ and $H_{1}^{\varangle}$, we also measured a correlation in $\cos \left(\Phi_{R}-\Phi_{\bar{R}}\right)$. This modulation is expected to vanish in pQCD, however, there are several non-perturbative processes that were proposed that could generate such an asymmetry. ${ }^{14}$ For example, QCD vacuum effects, such as local parity violating interactions with sphalerons or instantons could lead to a correlation in $\Phi_{R}-\Phi_{\bar{R}}$. As mentioned above, one of the main challenges in extracting these correlations at Belle, is that one cannot use spin asymmetries, thus it has to be shown that the acceptance of the detector, due to its fiducial volume or inactive areas, does not contribute to a false asymmetry. This check is done using Monte Carlo simulations that have been thoroughly vetted for a decade of Belle operations. In particular the $\cos \left(\Phi_{R}-\Phi_{\bar{R}}\right)$ asymmetry is sensitive to detector effects, since it is sensitive to repeating patterns in the acceptance. Other sources of false asymmetries are physics backgrounds like weak decays which are modeled well in the Belle Monte Carlo, using a combination of Pythia for the fragmentation and Evtgen for particle decays, or gluon radiation. To reduce false asymmetries coming from the acceptance and physics background we used a jet finding and reconstruction algorithm (anti$\mathrm{kT}$ with cone radius of 0.55 ) to suppress the effects of gluon radiation, a minimum energy per jet of $2.75 \mathrm{GeV}$ was required to reduce false asymmetries due to weak decays which leads to invisible energy carried away by neutrinos and a mixed event subtraction scheme was employed to reduce detector effects. A side effect of the energy cut is that the remaining sample consists only of dijets. With these cuts, false asymmetries in the simulation are compatible with zero.

Asymmetries $^{17}$ for the correlation corresponding to $G_{1}^{\perp}$ are very small and compatible with zero within the error bars. However, surprisingly the $\cos \left(\Phi_{R}-\Phi_{\bar{R}}\right)$ asymmetry corresponding to a possible strong local parity violating effect shows a signal the rises with the invariant mass. These results are still marked as "work in 
progress". However, they would indicate a correlation that is not expected in pQCD which would be extremely interesting.

\section{Belle II}

Belle stopped data taking in 2010 after collecting more than $1 \mathrm{ab}^{-1}$ of data, BaBar ceased operation in 2008 after recording abut $560 \mathrm{fb}^{-1}$. The data on tape from both experiments is sufficient for high precision measurements of FFs. However, there are areas on which a state of the art detector could improve, namely vertex detection and particle identification. Better vertex detection capabilities would allow a more efficient suppression of events where charm quark pairs are produced. Currently, the only efficient method to correct for charm contributions is to create charm enhanced samples by selecting events in which a $D^{0}$ or $D^{0 *}$ was produced, and then subtracting the charm asymmetries from the results obtained from the uncut sample. The disadvantage with this approach is that in particular the $D^{0 *}$ selection severely diminishes the available statistics and it selects a phase space for which the asymmetries are potentially different than they are for the whole sample, thus leading to a bias. On the particle identification side, BaBar was already achieving high kaon purities. However, the Belle PID system only achieved kaon purities of about $85 \%$, leading e.g. in channels with two identified kaons to purities only around $70 \%$. Therefore, as one moves forward to extract FFs for final states with one or more kaons, a better PID is essential. Both of these improvements are implemented in the Belle II detector which is scheduled to receive first beams for commissioning in 2016. In addition, Belle II will receive about 40x the luminosity that Belle had, allowing even more sensitive analysis differential in several variables at the same time. One example would be a measurement of the Collins FF simultaneously in $z$ and $k_{T}$. Belle II uses an imaging time of propagation detector (iToP) that achieves kaon purities of over $95 \%$ over most of the kinematic range ${ }^{18}$ and a vertex detector that allows to identify a significant fraction of charm events using the decay vertex.

\section{Conclusion}

While the measurement of the nucleon structure has made enormous progress over the last decade or so, experimentally and theoretically, the measurement of FFs still has to catch up. This is in particular of importance, since input on FFs is needed to analyze expected high precision data on the nucleon structure from SIDIS from the upcoming JLab $12 \mathrm{GeV}$ program. With the advent of the B-factories, highly precise measurements of fragmentation functions became possible and, for the first time, spin dependent fragmentation functions could be accessed directly. In recent years we have taken advantage of this data and have measured differential cross-sections for identified charged pions and kaons, from which the spin averaged FF $D_{1}^{q}$ can be extracted. Correlation measurements sensitive to the Collins FF for pions and kaons have been done as well as a measurement of the IFF $H_{1}^{\varangle}$. Ongoing measurements of $G_{1}^{\perp}$ which is only allowed to be nonzero in the TMD picture as well as a 
possible signal from nonperturbative QCD effects show that FF measurements can contribute significantly to our understanding of QCD. There is an active program ongoing to analyze the existing Belle data to extract, among others, the Collins FF for $\pi^{0}$ and $\eta$-mesons as well as to exract the intrinsic transverse momentum dependence of $D_{1}$. One motivation for the former, are the large asymmetries of $\pi^{0}$ transverse single spin asymmetries in $p p$ collisions and possibly even larger asymmetries for the $\eta$ meson. ${ }^{19}$ The latter is a crucial ingredient to extract the intrinsic transverse momentum of partons in the nucleon from SIDIS measurements.

\section{References}

1. V. Barone, A. Drago and P. G. Ratcliffe, Phys.Rept. 359, 1 (2002).

2. A. Bevan et al., The Physics of the B Factories, arXiv 1406.6311 (2014).

3. M. Leitgab et al., Phys.Rev.Lett. 111, p. 062002 (2013).

4. J. Lees et al., Phys.Rev. D88, p. 032011 (2013).

5. R. Sassot, Update from the DSS Fragmentation Function extraction (presented at the Indiana-Illinois Workshop on Fragmentation Functions, December 12-14, 2013).

6. J. C. Collins, Nucl.Phys. B396, 161 (1993).

7. A. Airapetian et al., Phys.Rev. D87, p. 012010 (2013).

8. F. Giordano, Precise spin-averaged and spin-dependent fragmentation function measurements at belle (Presented at DIS2014 - XXII. International Workshop on DeepInelastic Scattering and Related Subjects, 2014).

9. R. Seidl et al., Phys.Rev. D78, p. 032011 (2008).

10. J. Lees et al., Phys.Rev. D90, p. 052003 (2014).

11. D. Boer, R. Jakob and M. Radici, Phys.Rev. D67, p. 094003 (2003).

12. A. Vossen et al., Phys.Rev.Lett. 107, p. 072004 (2011).

13. A. Courtoy, A. Bacchetta, M. Radici and A. Bianconi, Phys.Rev. D85, p. 114023 (2012).

14. D. Boer, Back-to-back correlations of dihadron pairs (Presented at the Miniworkshop on Dihadron Fragmentation Functions (DiFF), Pavia, Sept 5-7, 2011, 2011).

15. J. C. Collins, S. F. Heppelmann and G. A. Ladinsky, Nucl.Phys. B420, 565 (1994).

16. J. C. Collins, Comment on 'Interference fragmentation functions and valence quark spin distributions in the nucleon', hep-ph/9801217 (1998).

17. A. Vossen, Di-hadron correlations in e+e- at Belle (presented at the Indiana-Illinois Workshop on Fragmentation Functions, December 12-14, 2013).

18. T. Abe et al., Belle II Technical Design Report, arXiv 1011.0352 (2010).

19. L. Adamczyk et al., Phys.Rev. D86, p. 051101 (2012). 